07.2

\title{
Нелинейность вольт-амперных характеристик однородных компенсированных детекторных структур из GaAs
}

\author{
(C) И.А. Прудаев ${ }^{1}$, М.Г. Верхолетов ${ }^{1,2}$ \\ ${ }^{1}$ Томский государственный университет, Томск, Россия \\ ${ }^{2}$ Институт нанотехнологий микроэлектроники РАН, Москва, Россия \\ E-mail: funcelab@gmail.com
}

Поступило в Редакцию 28 февраля 2019г.

В окончательной редакции 15 марта 2019г.

Принято к публикации 15 марта 2019г.

\begin{abstract}
Представлены результаты исследования процессов переноса носителей заряда и перезарядки глубоких уровней в полупроводниковых структурах для детекторов ионизирующих излучений. Исследованы арсенидгаллиевые структуры резистивного типа с барьерами Шоттки и с равномерным распределением глубокого акцептора хрома и глубокого донорного EL2-центра. Путем решения уравнений непрерывности и Пуассона с использованием коммерческого пакета проектирования обнаружен эффект обеднения объема детекторных структур по электронам с ростом поданного напряжения. Установлено, что нелинейность вольт-амперных характеристик структур обусловлена сменой типа проводимости при переходе от равновесного к неравновесному состоянию. При этом структуры с исходным (равновесным) дырочным типом проводимости имеют вольт-амперные характеристики, близкие к линейным.
\end{abstract}

Ключевые слова: детекторы ионизирующего излучения, арсенид галлия, глубокие уровни, транспорт носителей заряда.

DOI: 10.21883/PJTF.2019.11.47823.17760

Детекторы ионизирующего излучения на основе $\mathrm{GaAs}$ имеют ряд преимуществ по сравнению с широко используемыми детекторами на основе кремния [1-4], обладают более высокой эффективностью поглощения рентгеновского излучения с энергией квантов свыше $15 \mathrm{keV}$ и высокой радиационной стойкостью. Одна из задач, которая решается при разработке детекторов, связана с увеличением толщины чувствительного слоя, для чего используют однородные компенсированные структуры резистивного типа с омическими контактами или барьерами Шоттки. При этом компенсированный GaAs с высоким удельным сопротивлением $\left(10^{7}-10^{9} \Omega \cdot \mathrm{cm}\right)$ изготавливают либо за счет контролируемого введения глубоких донорных уровней EL2 в процессе роста слитков (первый подход $[3,4]$ ), либо путем компенсации глубоким акцептором - хромом - в процессе высокотемпературной диффузии (второй подход $[1,2]$ ).

Ранее установлено, что первый подход приводит к формированию стационарного домена сильного поля у катода детектора, что связано с сильной полевой зависимостью сечения захвата электронов EL2-центрами $[3,4]$. В итоге распределение напряженности поля становится неоднородным, а вольт-амперные характеристики $(\mathrm{BAX})$ - нелинейными. Экспериментальные результаты $[1,2,5]$ показали, что детекторные структуры, легированные $\mathrm{Cr}$, имеют более линейную BAX (иногда линейную в пределах малой погрешности измерений, не превышающей единиц процентов). По этой причине такие детекторы имеют преимущества, так как позволяют создать толстый чувствительный слой (до нескольких миллиметров) с однородным распределением напряженности электрического поля величиной $1-10 \mathrm{kV} / \mathrm{cm}[1]$.

Ранее не установлено, какие процессы приводят к нелинейности ВАХ и неоднородности электрического поля в структурах, компенсированных $\mathrm{Cr}$. Не установлены и технологические параметры, влияющие на степень нелинейности ВАХ. Имеющиеся аналитические модели описывают подобные структуры как последовательное соединение встречно включенных барьеров Шоттки со стороны анода и катода, а также объемного сопротивления компенсированного материала детектора [2,5]. Однако такое представление не учитывает особенностей перезарядки глубоких центров Cr и EL2, концентрация которых в детекторах достигает $10^{16}-10^{17} \mathrm{~cm}^{-3}$ [1-4]. В связи с этим в настоящей работе проведены расчеты протекания тока в детекторных структурах из GaAs, легированных $\mathrm{Cr}$ и EL2-центрами, с использованием численной модели.

В расчете геометрия всех структур оставалась постоянной: толщина $\mathrm{GaAs} 600 \mu \mathrm{m}$, площадь детекторных структур $10^{-6} \mathrm{~cm}^{2}$, катод и анод формировались барьерами Шоттки с высотой $\Phi_{b}=0.9 \mathrm{eV}$ относительно уровня Ферми в металле (решалась одномерная задача). Для всех структур задавалось однородное распределение концентраций мелкого донора $\left(N_{d}\right)$, глубокого донора $\left(N^{\mathrm{EL} 2}\right)$ и глубокого акцептора хрома $\left(N^{\mathrm{Cr}}\right)$.

В работе использовался пакет проектирования Sentaurus TCAD (Synopsys). Аналогично проведенным нами ранее исследованиям решалась система уравнений Пуассона и непрерывности с учетом перезарядки глубоких уровней $[6,7]$. Плотность тока задавалась гидроди- 


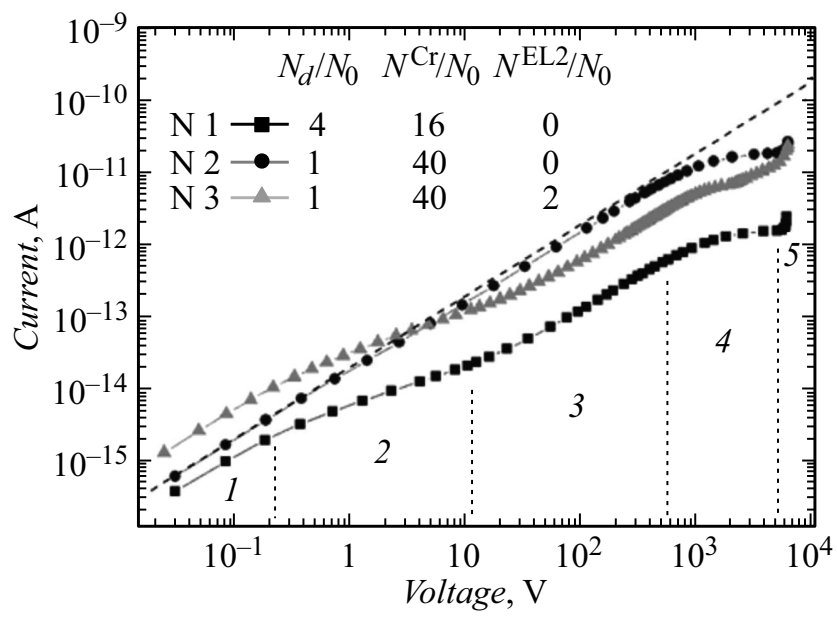

Рис. 1. Вольт-амперные характеристики трех детекторных структур с различным уровнем легирования. Номера структур представлены на рисунке, $N_{0}-$ константа нормирования. Штриховой прямой показана линейная зависимость силы тока от напряжения. Цифрами 1-5 указаны различные участки ВАХ для структуры № 1 .

намической моделью, в которой помимо диффузионного и дрейфового членов учитывался градиент температуры горячих носителей. В расчетах учитывались эффекты тепловой генерации тока через ловушки двух типов, так что темп генерации для каждой ловушки описывался следующим выражением:

$$
G=-\frac{N v_{n} v_{p} \sigma_{n} \sigma_{p} n_{i}^{2}}{v_{n} \sigma_{n}\left(n+n_{1}\right)+v_{p} \sigma_{p}\left(p+p_{1}\right)},
$$

где $N$ - концентрация ловушек (Cr или $\mathrm{EL} 2), v_{n}$ и $v_{p}-$ тепловые скорости электронов и дырок, $\sigma_{n}$ и $\sigma_{p}-$ сечения захвата электронов и дырок, $n$ и $p-$ концентрации электронов и дырок, $n_{i}$ - собственная концентрация носителей, $n_{1}$ и $p_{1}-$ концентрации носителей в случае совпадения уровня Ферми с уровнем ловушки.

Степень заполнения ловушек в стационарном состоянии определялась соотношением темпов захвата и выброса носителей заряда $[3,8]$. Эффекты лавинного умножения учитывались аналогично [6,7]. Параметры ловушки хрома взяты из работы [7] (энергия ионизации $0.79 \mathrm{eV}$, сечения захвата электронов и дырок составляют $3 \cdot 10^{-19}$ и $8 \cdot 10^{-17} \mathrm{~cm}^{2}$ соответственно). Энергия ионизации EL2-центров задавалась равной $0.75 \mathrm{eV}$, сечения захвата электронов и дырок в слабом поле для EL2-центров выбирались равными $5 \cdot 10^{-16}$ и $2 \cdot 10^{-18} \mathrm{~cm}^{2}$ соответственно [3]. Для EL2-центров полевая зависимость сечения захвата электронов задавалась при помощи модели, встроенной в пакет TCAD [8]. Данный подход позволил корректно смоделировать распределение напряженности электрического поля и ВАХ детекторных структур с EL2-центрами: аналогично работам $[3,4]$ в нашем расчете увеличение напряженности поля до $10 \mathrm{kV} / \mathrm{cm}$ приводило к росту сечения захвата электронов до $10^{-13} \mathrm{~cm}^{2}$ и формированию домена у катода в детекторе с EL2-центрами.

Результаты расчета ВАХ структур, легированных хромом и EL2-центрами, представлены на рис. 1. Условно все BAX можно разбить на пять участков: 1 - линейная зависимость $(U<0.1-1 \mathrm{~V}), 2-$ слабая сублинейная зависимость $(0.1-1<U<10-20 \mathrm{~V}), 3$ - линейная зависимость $(10-20<U<400-600 \mathrm{~V}), 4-$ участок насыщения тока $(400-600<U<5000 \mathrm{~V}), 5-$ участок резкого роста тока $(U>5000 \mathrm{~V})$. Участки 4 и 5 связаны с выходом на насыщение подвижности носителей заряда при росте напряженности поля и лавинным пробоем соответственно. Как правило, данные участки в эксперименте не наблюдаются (выходят за пределы режимов эксплуатации детекторов). Участки 1-3 практически всегда наблюдаются в экспериментах с разной степенью выраженности, за одним исключением: в эксперименте на участке 3 фиксируют слабую сверхлинейность [1,2,5], которая, на наш взгляд, может быть связана либо с эффектом разогрева детектора, либо с током утечки по поверхности.

Анализ результатов показал, что качественно механизмы формирования BAX для всех рассмотренных структур совпадают. Обсудим их подробно на примере структуры № 1 (рис. 1). На рис. 2 и 3 представлены профили распределения напряженности поля и концентрации носителей заряда для разных приложенных напряжений. Из рис. 2 видно, что поле проникает на всю область детектора уже при напряжении $U<10 \mathrm{~V}$. Вблизи контактов наблюдаются узкие области с повышенной напряженностью (ширина 0.15-0.2 $\mu \mathrm{m}$, напряженность $E=25-30 \mathrm{kV} / \mathrm{cm})$. Однако доля напряжения, приложенного к этим областям, не превышает $0.1 \%$ от полного напряжения, приложенного к детектору для $U<700 \mathrm{~V}$. Увеличение напряжения приводит к повышению относительной однородности напряженности поля в объеме детектора. Так, при $U=100 \mathrm{~V}$ отклонения

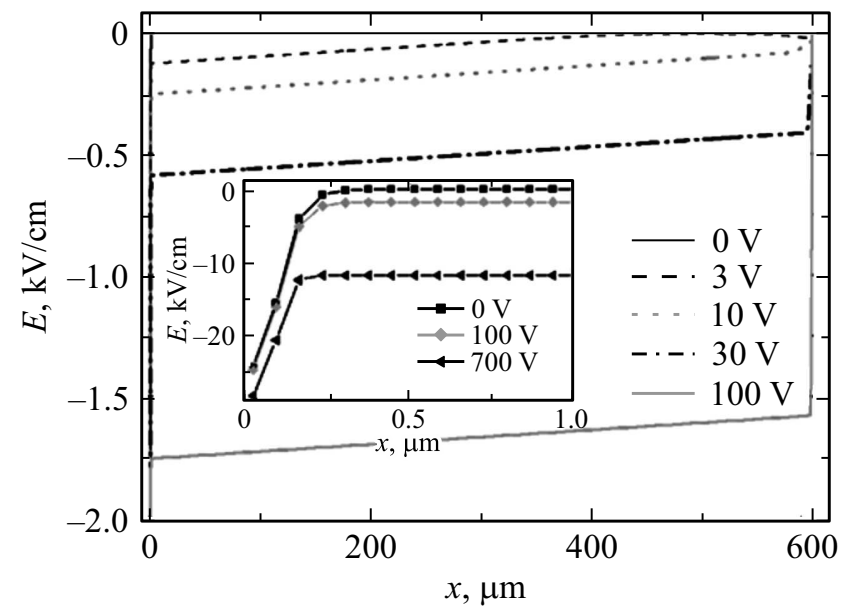

Рис. 2. Распределение напряженности поля в структуре № 1 при различных приложенных напряжениях (катод слева). На вставке показана приконтактная область в увеличенном масштабе. 

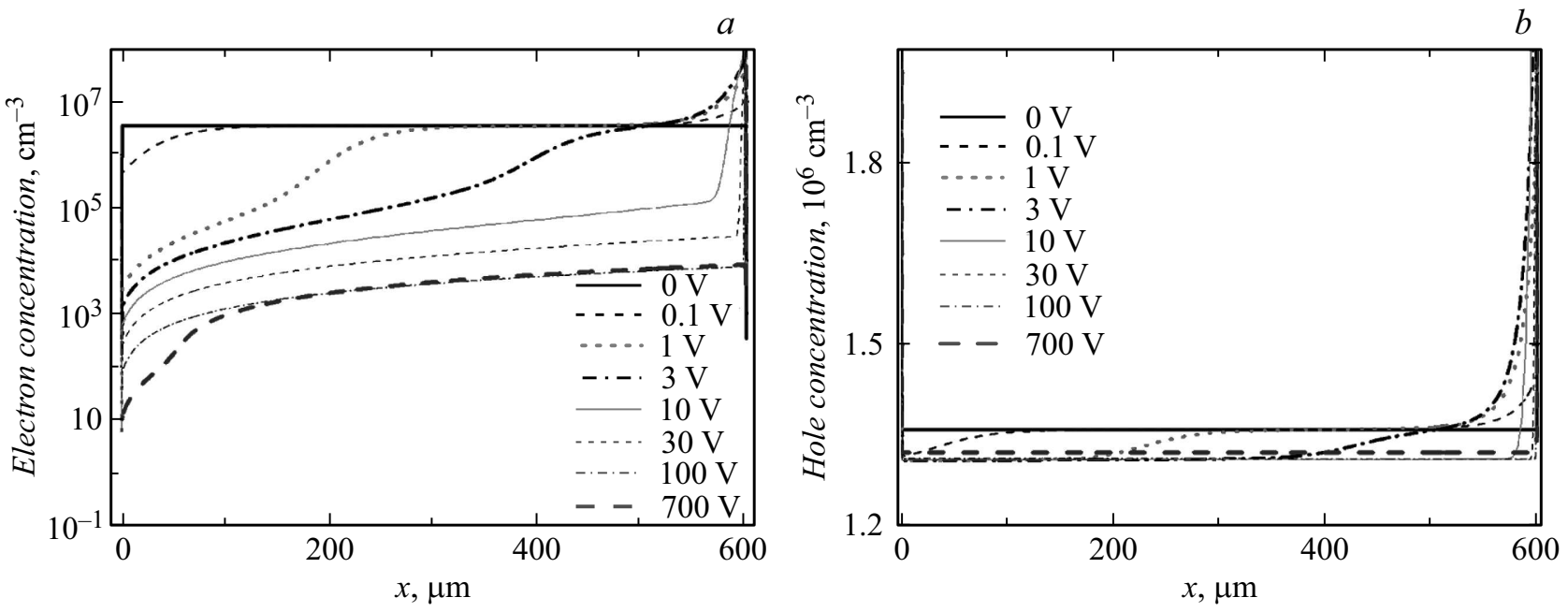

Рис. 3. Распределение концентрации электронов $(a)$ и дырок $(b)$ в структуре № 1 при различных приложенных напряжениях (катод слева).

напряженности составляют не более $10 \%$ от максимального значения (для $U=10 \mathrm{~V}$ не менее $70 \%$ ). Отметим, что увеличение концентрации хрома относительно концентраций мелкого донора и EL2-центров приводит к улучшению линейности ВАХ и однородности поля при фиксированном напряжении (рис. 1 и 2).

Из распределения концентрации носителей заряда (рис. 3) видно, что увеличение напряжения приводит к сильному обеднению структуры (концентрация электронов в объеме снижается на два-четыре порядка относительно равновесного значения). При этом концентрация дырок меняется слабо (уменьшается на 3-4\%). Данный эффект объясняется следующим образом. Барьеры Шоттки формируют у поверхности слой $p$-типа проводимости с концентрацией дырок $1.4 \cdot 10^{10} \mathrm{~cm}^{-3}$, что на четыре порядка превышает аналогичное значение в объеме. Данные носители возникают за счет тепловой генерации и высокой плотности состояний на поверхности, закрепляющей уровень Ферми ниже середины запрещенной зоны. Хотя приложение напряжения и приводит к дрейфу дырок в области поля, со стороны анода имеется бесконечный источник, поддерживающий постоянный уровень их концентрации, - инжектирующий слой $p$-типа. С другой стороны, источника электронов в рассмотренной структуре нет, что приводит к выносу электронов из области поля и обеднению детектора по этому типу носителей.

Представленные результаты показывают, что нелинейность ВАХ, как и неоднородность распределения напряженности поля, наблюдается в структурах с наибольшей концентрацией равновесных электронов. Если в структуре исходный тип проводимости является электронным (в равновесии), то обеднение по электронам приводит к инверсии типа проводимости объема и появлению сублинейного участка на ВАХ (структуры № 1 и 3). Если же в состоянии равновесия исходный тип проводимости является дырочным (структура № 2), то обеднение по электронам при увеличении поданного напряжения не сказывается на проводимости детектора в неравновесных условиях. В этом случае ВАХ приближается к линейному виду (штриховая прямая на рис. 1), а напряженность электрического поля распределяется однородно в объеме детектора при малых приложенных напряжениях (единицы вольт).

Отметим, что полученный результат принципиально отличается от представлений о наличии барьеров Шоттки, ограничивающих ток в детекторных структуpax [5]. Область пространственного заряда такого барьеpa, созданная ионизированными донорами, практически не расширяется при приложении напряжения, так как в неравновесных условиях проводимость обусловлена дырками (для материала $p$-типа барьер приводит к обогащению по основным носителям - дыркам). Распространение поля в глубь детектора при увеличении напряжения контролируется балансом между темпами захвата и выброса носителей с ловушек, а плотность положительного заряда в объеме оказывается на несколько порядков ниже концентрации положительно заряженных ионов в приконтактном слое барьера Шоттки.

\section{Финансирование работы}

Работа выполнена при поддержке Министерства образования и науки РФ (государственное задание № 11.2247.2017).

\section{Конфликт интересов}

Авторы заявляют, что у них нет конфликта интересов. 


\section{Список литературы}

[1] Tyazhev A.V., Budnitsky D.L., Koretskay O.B., Novikov V.A., Okaevich L.S., Potapov A.I., Tolbanov O.P., Vorobiev A.P. // Nucl. Instr. Meth. A. 2003. V. 509. P. 34-39.

[2] Veale M.C., Bell S.J., Duarte D.D., French M.J., Schneider A., Seller P., Wilson M.D., Lozinskaya A.D., Novikov V.A., Tolbanov O.P., Tyazhev A., Zarubin A.N. // Nucl. Instr. Meth. A. 2014. V. 752. P. 6-14.

[3] Rogalla M., Runge K. // Nucl. Instr. Meth. A. 1999. V. 434. P. 44-56.

[4] Cola A., Reggiani L., Vasanelli L. // Semicond. Sci. Technol. 1997. V. 12. P. 1358-1364.

[5] Kolesnikova I., Lozinskaya A., Mihaylov T., Novikov V., Shemeryankina A., Sherbakov I., Tolbanov O., Tyazhev A., Zarubin A. // JINST. 2016. V. 11. P. C03059 (1-6).

[6] Прудаев И.А., Верхолетов М.Г., Королёва А.Д., Толбанов О.П. // Письма в ЖТФ. 2018. Т. 44. В. 11. С. 21-29.

[7] Prudaev I.A., Oleinik V.L., Smirnova T.E., Kopyev V.V., Verkholetov M.G., Balzovsky E.V., Tolbanov O.P. // IEEE Trans. Electron Dev. 2018. V. 65. N 8. P. 3339-3344.

[8] Sentaurus Device User Guide.

www.sentaurus.dsod.pl/manuals/data/sdevice_ug.pdf 